\title{
Low-luminosity radio-loud active galactic nuclei
}

\author{
Gabriele Giovannini ${ }^{* 1,2}$ and Marcello Giroletti ${ }^{2}$ \\ ${ }^{1}$ Astronomy Department - Bologna University \\ via Ranzani 1, 40127 Bologna, Italy \\ ${ }^{2}$ INAF - Istituto di Radioastronomia \\ via Gobetti 101, 40129 Bologna, Italy \\ E-mail: ggiovanndira.inaf.it, girolettidira.inaf.it
}

I review observational properties of low-power radio-loud AGN. High-resolution VLBI observations allow to estimate the jet velocity and orientation with respect to the line of sight and the determination of the Doppler factor. These data reveal rich structures, including two-sided jets and secondary components. New results on $1144+35$, a giant radio source with superluminal motion, are shown in detail.

The 8th European VLBI Network Symposium

September 26-29, 2006

Toruń, Poland

${ }^{*}$ Speaker. 


\section{Introduction}

The study of parsec-scale properties of radio galaxies is crucial to derive physical properties of the central regions of the Active Galactic Nuclei (AGN) and provides the observational basis of Unified Models. A comparison of pc- and kpc-scale morphology and properties is the key to understand the origin and evolution of extended radio sources.

To investigate the properties of radio galaxies at pc resolution it is important to select sources from low-frequency radio catalogues, where the source properties are dominated by the unbeamed extended emission and are not affected by observational biases related to orientation effects. To this end, we undertook a project of observations of a complete sample of radio galaxies selected from the B2 and 3CR catalogues with $z<0.1$ (i.e. no constrain on the core flux density): the Bologna Complete Sample (BCS [6]). The sample consists of 95 sources. At present, 60 out of 95 sources have been studied with VLBI observations. As a first step, we observed sources with an arcsecond core flux density $\sim 10 \mathrm{mJy}$ or more at $5 \mathrm{GHz}$ and now we are observing sources with a core flux density in the range 1 to $10 \mathrm{mJy}$ (see e.g. Fig. 1) using phase referencing.

\section{Sources morphology}

Parsec-scale structures are mostly one-sided because of relativistic Doppler boosting effects, however a large number of sources with two-sided jets have been found.

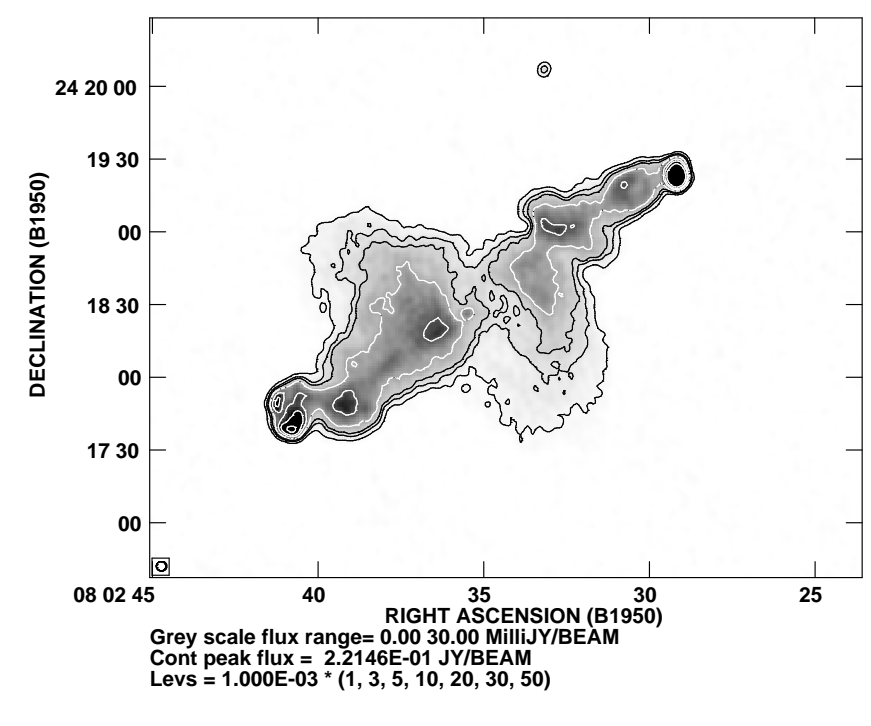

a. VLA

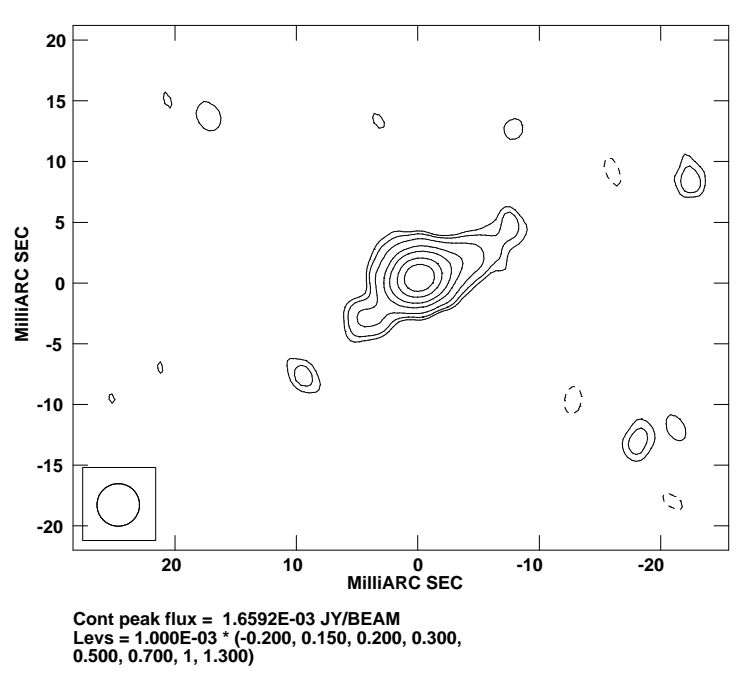

b. VLBI.

Figure 1: VLA image of the kpc-scale structure of 3C192 from http://www.jb.man.ac.uk/atlas (a). Phasereferenced VLBA image of the core of 3C192 (b).

Among observed sources, 18 ( $\sim 30 \%)$ show a two-sided pc-scale structure. We note that in previous surveys of large samples selected at high frequency, as the Caltech survey, the percentage of two-sided structures is only of $4.6 \%$. The difference between the percentage of symmetric sources, in the present sample and in previous samples is naturally explained in the framework of 
unified scheme models by the fact that present sources have been selected at low frequency, show relatively faint cores, and are therefore less affected by orientation bias. In the majority of sources, we find a good agreement between the orientation of the pc- and kpc-scale structures. This result supports the notion that large distortions detected in BL Lac sources are due to small intrinsic bends amplified by the small angle of the BL Lac jets with respect to the line of sight.

\section{Jet kinematics}

\subsection{Proper motion}

Many AGNs contain compact radio sources with different components that appear to move apart. Multi-epoch studies of these sources provide a direct measure of the apparent jet pattern velocity $\left(\beta_{a} c\right)$. From the measure of $\beta_{a}$ we can derive constraints on $\beta_{p}$ and $\theta$, where $\beta_{p} c$ is the intrinsic velocity of the pattern flow and $\theta$ is the jet orientation with respect to the line of sight:

$$
\beta_{p}=\beta_{a} /\left(\beta_{a} \cos \theta+\sin \theta\right)
$$

The main problem is to understand the difference between the bulk and pattern velocity. In a few cases, where proper motion is well defined and the bulk velocity is strongly constrained, there is a general agreement between the pattern velocity and the bulk velocity (see e.g. NGC 315 [1], and $1144+35$, here). However, in the same source, we can have different pattern velocities as well as stationary and high-velocity moving structures. Moreover, we note that in many well-studied sources, the jet shows a smooth and uniform surface brightness and no (or very small) proper

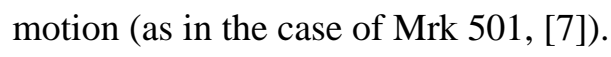

\subsection{Bulk velocity}

Assuming that the jets are intrinsically symmetric, we can use relativistic effects to constrain the jet bulk velocity $\beta c$ and orientation with respect to the line of sight $(\theta)$, as discussed e.g. in [5]. I will discuss here only the jet - counter-jet (c-j) brightness ratio and the core dominance since they are the most used methods.

- Assuming that the jets are intrinsically symmetric, we can use the observed jet to c-j brightness ratio $\mathrm{R}$ to constrain the jet bulk velocity $\beta c$ and its orientation with respect to the line of sight:

$$
R=(1+\beta \cos \theta)^{2+\alpha}(1-\beta \cos \theta)^{-(2+\alpha)}
$$

where $\alpha$ is the jet spectral index $\left(\mathrm{S}(v) \propto v^{-\alpha}\right)$.

- At arcsecond resolution, the core radio emission measured at $5 \mathrm{GHz}$ is dominated by the Doppler-boosted pc-scale relativistic jet. The source radio power measured at low frequency (e.g. $408 \mathrm{MHz}$ ), instead, is due to the extended emission, which is not affected by Doppler boosting. At low frequency, the observed core radio emission is not relevant since it is mostly self-absorbed. Given the existence of a general correlation between the core and the total radio power discussed in [ [ ], we can derive the expected intrinsic core radio power from the 
unboosted total radio power using the estimated best-fit correlation (continuum line in Fig. 2):

$$
\log P_{c}=(0.62 \pm 0.04) \log P_{t}+(7.6 \pm 1.1)
$$

The comparison between the expected intrinsic and the observed core radio powers will give constraints on the jet velocity and orientation ([4]).

The large dispersion in the core radio power visible in Fig. 2 is expected because of the strong dependence of the observed core radio power on $\theta$ and $\beta$.

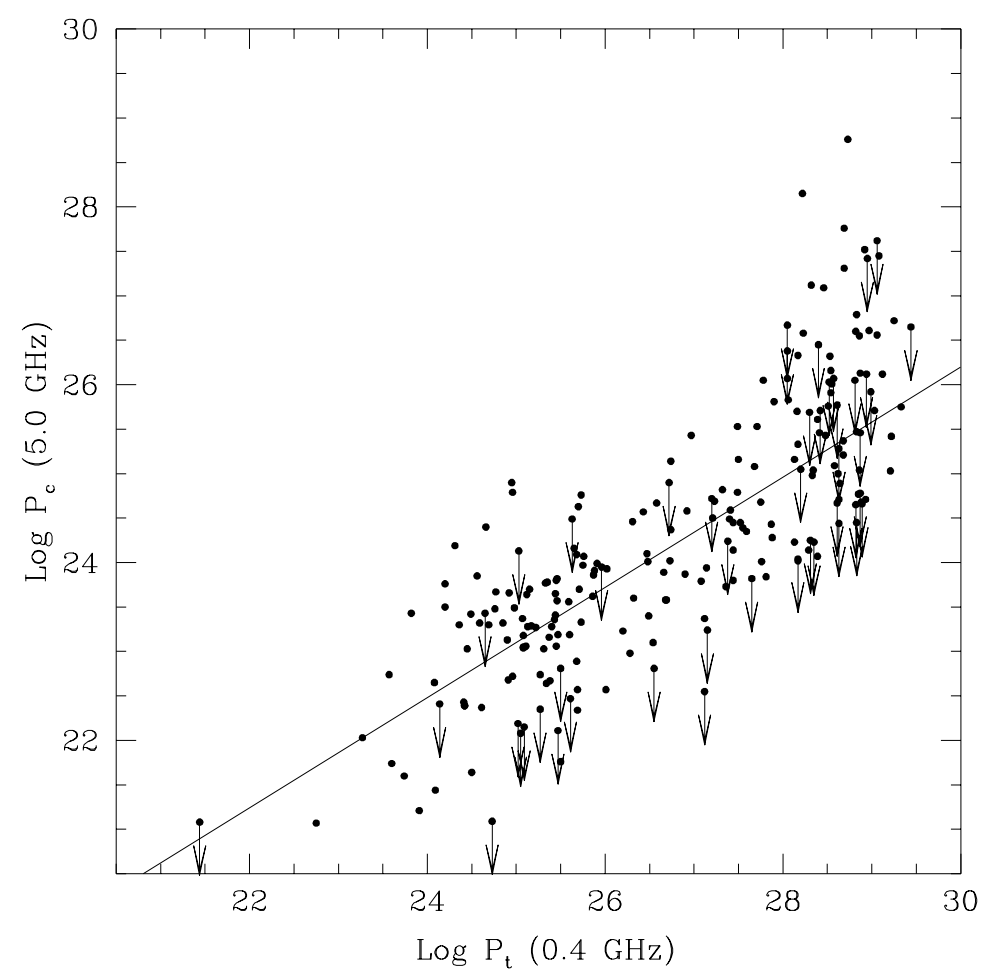

Figure 2: Arcsecond core radio power at $5 \mathrm{GHz}\left(\mathrm{P}_{c}\right)$ versus total radio power at $0.4 \mathrm{GHz}\left(\mathrm{P}_{t}\right)$ for $\mathrm{B} 2$ and $3 \mathrm{CR}$ radio sources (see [2]). Arrows are upper limits when a nuclear emission was not detected. The line is the best fit of the data corresponding to a source orientation with respect to the line of sight of $60^{\circ}$.

From the data dispersion, assuming that no selection effect is present in the source orientation $\left(\theta=0^{\circ}\right.$ to $\left.90^{\circ}\right)$, we can derive that the jet Lorentz factor $\Gamma$ has to be in the range 3 to 10 , otherwise we should observe a smaller or larger core radio power dispersion.

\section{Results}

To derive statistical properties of radio jets on the pc-scale, we used all observational data for the 60 sources in our sample with VLBI data available. We found that in all sources the pc-scale jets move at high-velocity. No correlation has been found between the jet velocity and the core 


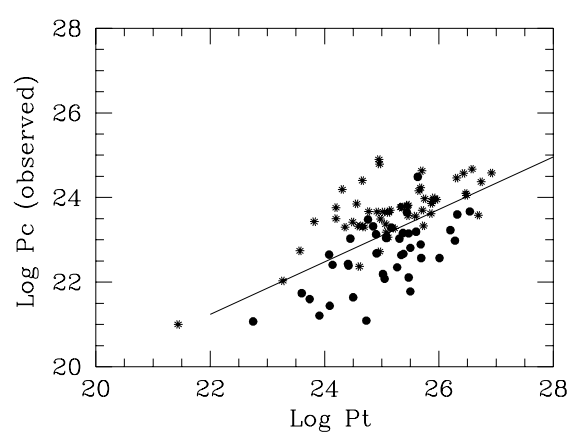

a. observed data

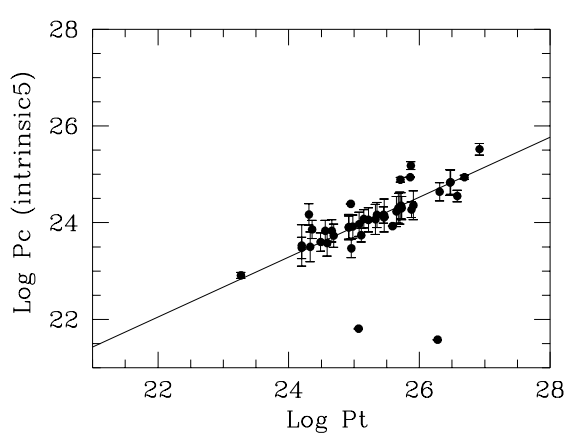

b. intrinsic data.

Figure 3: Observed core radio power versus total radio power for sourc es from the BCS with VLBI data (a). As (a) but with intrinsic core radio power versus total radio power (b).

or total radio power. Highly relativistic parsec-scale jets are present regardless of the radio source power. Sources with a different kpc-scale morphology and total radio power have pc-scale jets moving at similar velocities.

We used the estimated jet $\beta$ and $\theta$ to derive the Doppler factor $\delta$ for each source, and the corresponding intrinsic core radio power (assuming $\alpha=0$ ):

$$
P_{c-\text { observed }}=P_{c-\text { intrinsic }} \times \delta^{2}
$$

We found a good correlation between $\mathrm{P}_{c-\text { intrinsic }}$ and $\mathrm{P}_{t}$ with a small dispersion, since plotting $\mathrm{P}_{c-\text { intrinsic }}$ we removed the spread due to the different orientation angles (Fig. 3). These results are in agreement with the expectations from unified models.

\section{Low-power radio cores}

The previous results are supported by the studies of many sources. However, when studying sources with a faint arcsecond-scale radio core, never studied before in a large sample, we have a relatively large number of peculiar pc-scale structures, for which interp retation and connection with the large-scale structure is not clear. The low number of sources studied so far does not allow to distinguish between a general property of sources with a mJy (or lower) radio core and the presence of a few peculiar sources, with possible signatures of restarted activity.

Here, we present the radio image of $3 \mathrm{C} 310$ at kpc and pc resolution. A more detailed discussion will be presented and discussed in Giovannini et al. (in preparation), together with other peculiar structures in our sample (e.g. 1346+26 and 0836+29B). At kpc scale, 3C310 is an extended, relaxed double source with a bright core and a short jet in $\mathrm{N}$ direction (see Fig. 4). The lobes show a filamentary structure. Moreover, the total flux-density measures from the NED database show that $3 \mathrm{C} 310$ has a steep radio spectrum $(\alpha>1)$. These two facts suggest that the radio lobes are old, but a detailed study is not available in the literature yet.

As noted by [9] for the optical image, this galaxy is flattened east-west, in an almost perpendicular direction to the kpc radio-jet axis. VLBI images (see also [8]) show an extended structure elongated perpendicularly to the kpc-scale radio structure and to the short jet visible in the VLA 


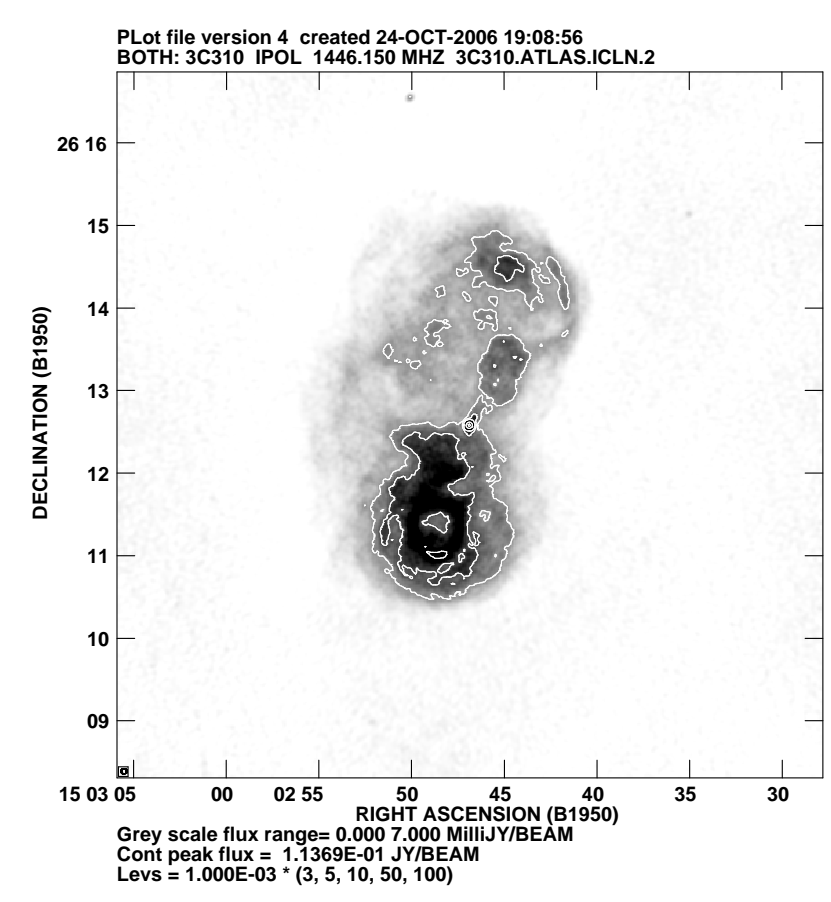

Figure 4: VLA arcsecond-scale radio image of 3C310 from http://www.jb.man.ac.uk/atlas.

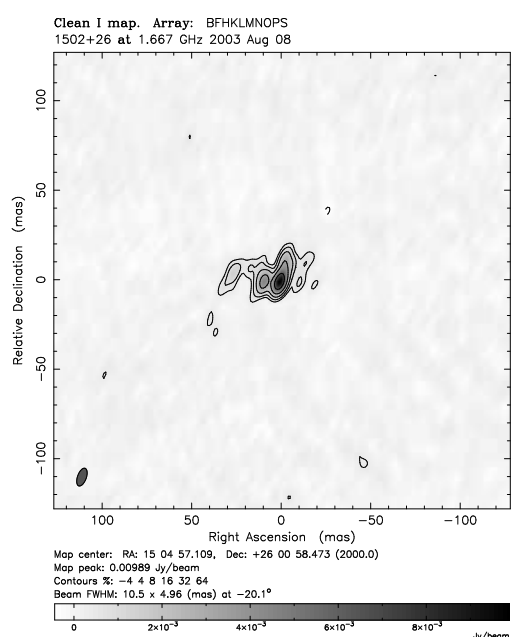

a. VLBI image at $1.4 \mathrm{GHz}$

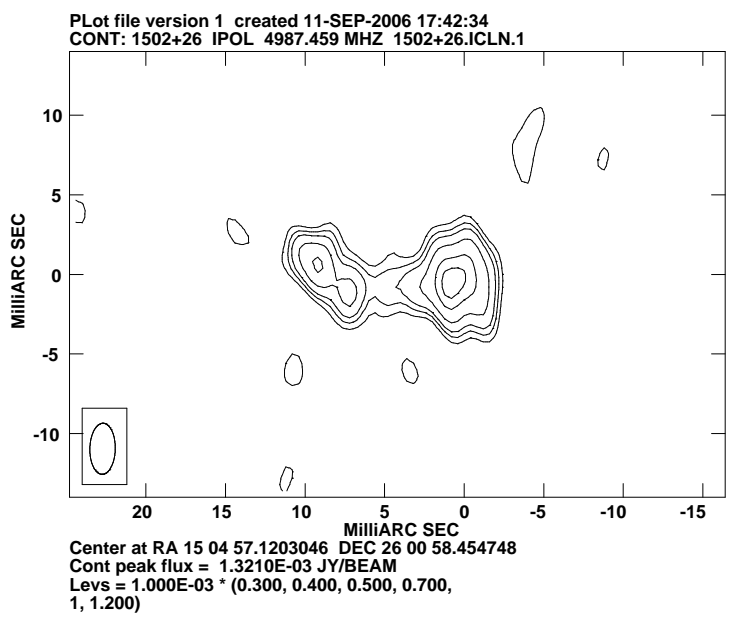

c. VLBI image at $5 \mathrm{GHz}$.

Figure 5: Phase-referenced VLBI images at $1.4 \mathrm{GHz}(\mathrm{a})$ and at $5 \mathrm{GHz}$ (b) of 3C310.

image (see Fig. 5). The pc-scale structure is very peculiar since a dominant core structure is not visible even at $5 \mathrm{GHz}$. With present data it is not possible to identify a core emission. Moreover, the extended emission is resolved and looks quite different from most pc-scale jets detected in radio galaxies. The low brightness and the absence of evident boosting effects suggest an orientation near to the plane of the sky, but in this case the large difference in the position angle of the pc- and kpc-scale structures has to be real and not amplified by projection effects. More data at different resolution are necessary to understand the pc-scale structure and its connection with the kpc-scale 
radio lobes.

\section{6. $1144+35$}

The low-power radio source B2 $1144+35 \mathrm{~B}$ was identified with a faint $\left(\mathrm{m}_{p g}=15.7\right) \mathrm{Zwicky}$ galaxy (Zw186.48) in a medium-compact galaxy cluster at a redshift of 0.0630 . From the radio point of view, $1144+35$ has a peculiar structure as discussed in detail by [3]. New observations confirm the general structure discussed in [3]. The pc-scale structure (Fig. 6a) is resolved in a nuclear source (C) with a short jet (D) and counter-jet structure (E). At about 25 mas from the core, we have an extended jet-like structure (A and B components), which is the dominant structure in the VLBI images. This jet structure is extended, clearly limb-brightened and connected to the core by a low-brightness emission.

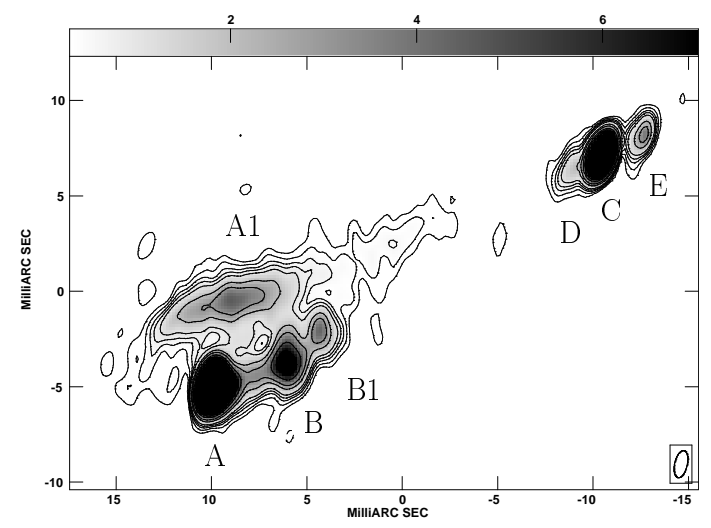

a. VLBI at $8.4 \mathrm{GHz}$

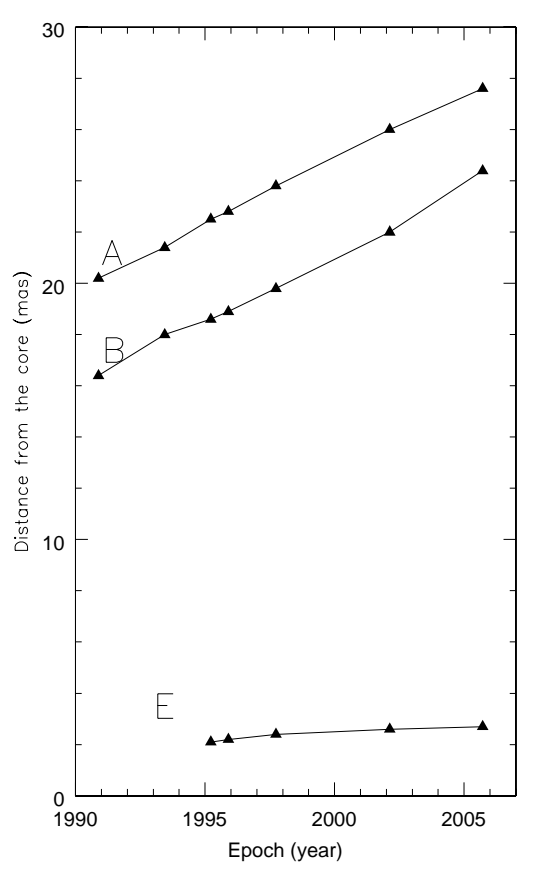

b. Proper Motion.

Figure 6: VLBI image of the pc-scale structure of 1144+35 (a). Core distance of components A, B, and E at different epochs (b).

We used the observations made at different epochs to measure the apparent proper motion of components $\mathrm{A}, \mathrm{B}$, and $\mathrm{E}$ with respect to the core component $\mathrm{C}$. In Fig. 6b, we show the distance of components A, B, and $\mathrm{E}$ from $\mathrm{C}$ at different epochs. From these data, we measured for the first time a proper motion of the counter-jet (E) structure (in agreement with the previous upper limit): $\beta_{a-c j}=0.23$, while for components $\mathrm{A}$ and $\mathrm{B}$ we confirm (note the different cosmology used here with respect to previous papers): $\beta_{a-j}=1.92$.

Since we know the source angular distance (D) in the present cosmology, we can also derive the intrinsic jet orientation [10]:

$$
D=0.5 \operatorname{ctan} \theta\left(\mu_{a}-\mu_{r}\right)\left(\mu_{a} \mu_{r}\right)^{-1}
$$




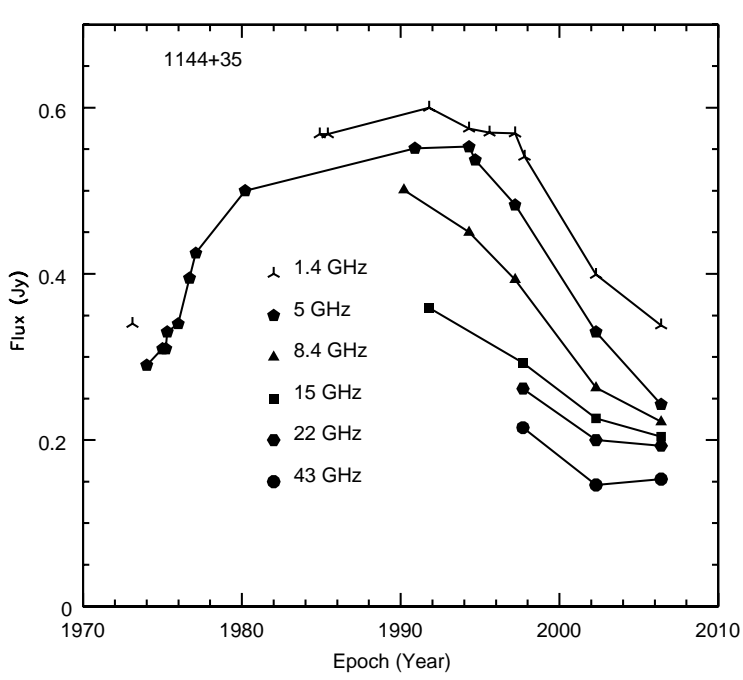

a. Arcsecond core

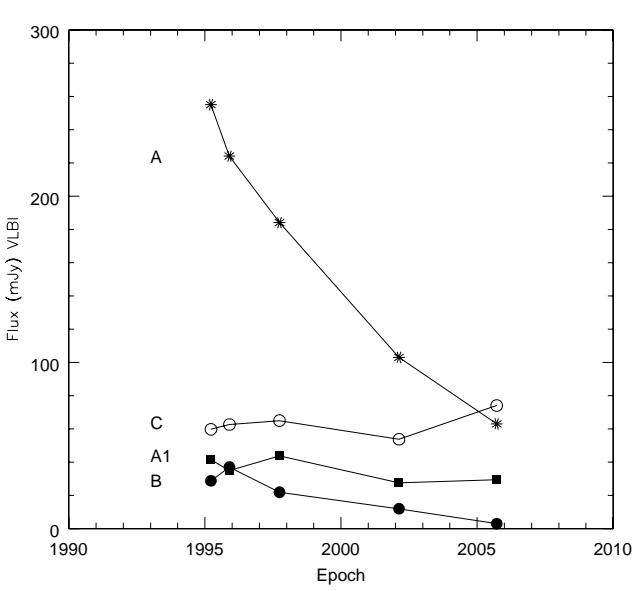

b. VLBI components.

Figure 7: Flux-density measures of the 1144+35 arcsecond core at different epochs and frequencies (a). 8.4-GHz flux density measures of components A, A1, B, and C at different epochs (b).

where $\mu_{a}$ and $\mu_{r}$ are the angular velocities of the approaching (a) and receding (r) jet, respectively.

We find $\theta=33^{\circ}$ and therefore $\beta=0.94$ in agreement with the measured jet - counter-jet arm ratio $\sim 10$ which implies $\beta \cos \theta \sim 0.82$ with the possible values: $\beta=0.95, \theta=30^{\circ}$. The morphology of this source suggests a recurrent radio activity. We have:

- the Mpc-scale (the oldest) structure,

- the naked jets and the dominant core on the arcsecond-scale,

- the VLBI structure discussed here.

Assuming a constant jet velocity, the main VLBI jet structure was emitted from the core about year 1950. One more piece of evidence of a recurring activity comes from the arcsecond core and mas-structure flux-density variability (Fig. 7). From the comparison of the light curves of the arcsecond-scale core and the mas-scale components, it is clear that: 1) the arsecond-scale variability in not due to the mas core $\mathrm{C}$ but to the main jet component (A); 2) in the last few years (after 2002), the flux-density of the mas core $\mathrm{C}$ is increasing and this increase of the flux-density is visible also thanks to the arcsecond core monitoring, but only at high frequency.

A possible explanation is that after 2002 and before of 2006 the core $C$ started a new active phase and a new component is emerging. This new component is not yet visible in our images and it is still self-absorbed at frequencies lower than $8.4 \mathrm{GHz}$, which implies, in equipartition conditions, 
a size of this new component of about 0.03 mas. If this component is moving at the same velocity as the main jet, we should start to see it in VLBI images at $8.4 \mathrm{GHz}$ in about 2 years.

\section{Conclusions}

- The pc-scale jet morphology is the same in high- (FR II) and low- (FR I) power sources; the pc-scale morphology is in agreement with expectations from unified models.

- There is a good agreement between the pc- and kpc-scale orientation.

- The pc-scale jet velocity is highly relativistic in FR II and FR I sources. It is not related to the total or core radio power of the source. No correlation was found with the kpc-scale structure.

- In some sources with a low-power nuclear source, we find a peculiar morphology: restarted activity and a complex mas-scale structure not yet understood, misaligned with the kpc-scale structure.

- The low-power source $1144+35$ shows a superluminal motion and a restarted activity. The core flux density is variable and an increase of the core flux density at high frequency suggests the presence of a new component that could be visible in VLBI images in 2007-2008.

\section{References}

[1] W.D. Cotton, L. Feretti, G. Giovannini, L. Lara, T. Venturi 1999, ApJ 519, 108

[2] G. Giovannini, L. Feretti, L. Gregorini, P. Parma 1988, A\&A 199, 73

[3] G. Giovannini, G.B. Taylor, E. Arbizzani, M. Bondi, W.D. Cotton, L. Feretti, L. Lara, T. Venturi 1999, ApJ 522, 101

[4] G. Giovannini, W.D. Cotton, L. Feretti, L. Lara, T. Venturi 2001, ApJ 552, 508

[5] G. Giovannini 2004, Ap\&SS 293, 1 [astro-ph/ 0406116 ]

[6] G. Giovannini, G.B. Taylor, L. Feretti, W.D. Cotton, L. Lara, T. Venturi 2005, ApJ 618, 635

[7] M. Giroletti, G. Giovannini, L. Feretti, W.D. Cotton, et al. 2004, 600, 127

[8] N.A.B. Gizani, M.A. Garrett 2002, in 6th European VLBI Network Symposium, held in Bonn, June 25th-28th 2002, proceedings edited by E. Ros, R. W. Porcas, A. P. Lobanov, and J. A. Zensus, published by the Max-Planck-Institut fuer Radioastronomie (Bonn). p. 159.

[9] A.R. Martel, S.A. Baum, W.B. Sparks, EM. Wyckoff, et al. 1999, ApJS 122, 81

[10] I.F. Mirabel, L.F. Rodriguez 1999 ARA\&A 37409 